\title{
Parasomnias and obstructive sleep apnea syndrome: in search for a parasomnia evaluating tool appropriate for OSAS screening
}

TOMASZ WIECZOREK ${ }^{1, A-F}$, MACIEJ LORENC ${ }^{2, A-D}$, HELENA MARTYNOWICZ ${ }^{3, A, B, E, ~ F, ~}$

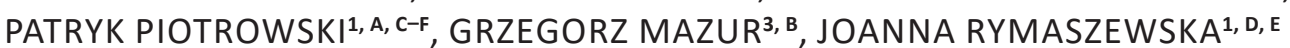

${ }^{1}$ Department and Clinic of Psychiatry, Wroclaw Medical University, Poland

2 Psychiatric Ward, Alfred Sokolowski Specialized Hospital, Walbrzych, Poland

${ }^{3}$ Department and Clinic of Internal Medicine, Occupational Diseases, Hypertension and Clinical Oncology, Wroclaw Medical University, Poland

A - Study Design, B - Data Collection, C - Statistical Analysis, D - Data Interpretation, E - Manuscript Preparation, F - Literature Search, G - Funds Collection

Summary Background. Obstructive sleep apnea syndrome (OSAS) is the most common form of sleep-disordered breathing. It is also a known major risk factor of parasomnias.

Objectives. To verify if an additional diagnostic tool for parasomnia assessment might be useful in OSAS screening. Additionally, to investigate the possible relations of parameters measured during polygraphy evaluation and the severity/frequency of parasomnia episodes in order to find the pathophysiological background of increased frequency of parasomnias in OSAS cases.

Material and methods. Parasomnia evaluation with the Paris Arousal Disorder Severity Scale (PADSS) and OSAS evaluation with polygraphy in a group of 105 adult patients hospitalized for assessment of possible OSAS.

Results. According to the polygraphic evaluation, patients were divided into: non-OSAS ( $n=19,18.1 \%)$, mild OSAS $(n=31,29.52 \%)$, moderate OSAS ( $n=21,20 \%$ ) and severe OSAS ( $n=34,32.38 \%)$. Based on the recommended cut-off score of 13 points in PADSS, two groups of patients emerged after the parasomnia evaluation: with positive $(n=10)$ and negative $(n=95)$ results. PADSS achieved sensitivity of $10.9 \%$ and $92 \%$ specificity when used for moderate and severe OSAS screening. The positive predictive value (PPV) reached $60 \%$, negative predictive value (NPV) $-48.4 \%$. A statistically significant elongation of the percentage of estimated sleep time below $80 \%$ blood oxygen saturation emerged in patients with positive PADSS scores $(p=0,006)$. Focusing on the parasomnia frequency criterion did not significantly affect the results.

Conclusions. Due to low sensitivity, PPV and NPV PADSS cannot be used as a screening tool in possible OSAS assessment. Another too with higher sensitivity should be developed, also including assessment of other parasomnias and sleep-related movement disorders. Cyclic intermittent hypoxia might be the cause of the higher frequency of arousal disorders in OSAS patients. Key words: parasomnias, sleep apnea syndromes, sleep apnea, obstructive.

Wieczorek T, Lorenc M, Martynowicz H, Piotrowski P, Mazur G, Rymaszewska J. Parasomnias and obstructive sleep apnea syndrome: in search for a parasomnia evaluating tool appropriate for OSAS screening. Fam Med Prim Care Rev 2018; 20(2): 176-181, doi: https:// doi.org/10.5114/fmpcr.2018.76464.

\section{Background}

Obstructive sleep apnea syndrome (OSAS) is the most common, but still underestimated, form of sleep-disordered breathing, characterized by the collapse of the upper airway leading to intermittent arterial oxygen desaturation, micro arousal-related deregulation of the sympathetic nervous system, oxidative stress, and chronic inflammation [1, 2], excessive daytime sleepiness causing automobile accidents and impaired functioning, among other symptoms [3].

One out of five adults suffers from at least a mild degree of OSAS, and the prevalence in adults 30-69 years of age is estimated at $17 \%$, and $41-58 \%$ in obese patients. It was demonstrated that that $93 \%$ of females and $82 \%$ of males with moderate to severe OSAS had not been clinically diagnosed, despite access to healthcare [4-6]. OSAS has been independently associated with hypertension [7], stroke [8, 9], myocardial ischemia [10, 11], dyslipidemia $[12,13]$, impaired glucose tolerance $[14,15]$, and arrhythmias with an increased risk for sudden cardiac death [16].

OSAS is often the cause of impaired daytime functioning. Its symptoms include: daytime sleepiness, morning headache, attention disorders, impaired memory, decreased libido, affective disorders, fear of going to bed, snoring, increased sweating, heart palpitations, and dryness of the oral cavity during sleep and in the morning [17-19].

Nowadays, the diagnostic gold standard of OSAS is polysomnographic evaluation (PSG) - OSAS is classified based on the Apnea-Hypopneas Index (AHI), which describes the number of those nocturnal events per hour of sleep. According to the American Academy of Sleep Medicine, OSAS is classified as mild $(\mathrm{AHI}>5)$, moderate $(\mathrm{AHI}>15)$ or severe $(\mathrm{AHI}>30)$ [20]. However, in this study only the polygraphic record without EEG registration was performed, due to technical limitations. Nevertheless, polygraphy was sufficient to determine basic respiratory parameters during sleep.

The occurrence of psychopathological symptoms in OSAS patients (mainly of moderate or severe level) suggests the possible cooperation of specialists in different fields of internal medicine, specialists and researchers with psychiatrists in order to improve the quality and effectiveness of diagnostic and therapeutic procedures.

OSAS is also a major risk factor of parasomnias [21-23]. Parasomnia is defined as undesirable physical or experiential 
events that accompany sleep $[21,24,25]$. They usually include abnormal motor activity, perceptions, emotions, autonomic nervous system dysfunctions and dreaming [21]. They can be disorders of arousal but also of sleep-stage transition and sleep/ /awakening events $[22,23]$. Some of them are known to occur only in a specific sleep stage. NREM parasomnias include confusional arousals, sleepwalking and sleep terrors. Conversely, REM sleep behavior disorder (RBD), nightmares and isolated sleep paralysis emerge from the REM stage. Other parasomnias (like enuresis, sleep talking or exploding head syndrome) may occur during different stages or at sleep/awakening transition [25]. Catathrenia was also included in this group of disorders in the International Classification of Sleep Disorders $2^{\text {nd }}$ edition (ICSD$2)$, but in the $3^{\text {rd }}$ edition it was moved to the group of Sleeprelated Breathing Disorders (SBD) [24, 25].

Many studies have so far presented pathophysiological theories explaining the causes of these events. Most parasomnias may result from incomplete dissociation of wakefulness, NREM and REM sleep [22, 26, 27, 39]. Sleep is not a global process, and does not manifest in the same way in different brain parts. Parasomnia might occur when two different sleep stages overlap or take place simultaneously. But this process is sometimes insufficient for disorders to occur - several studies state that other factors contribute to arousal events, especially including motor activity [23]. Those factors include: activation of locomotor centers (cortical and thalamocingular pathways [28, 29]), sleep inertia [30,31] and sleep state instability (cyclic alternating pattern, representing the NREM instability) [32-34]. It is presumed that comorbidities leading to cortical arousals, promoting sleep inertia and affecting sleep microstructure (like OSAS and sleeprelated movement disorders) may lead to intrusions of some sleep/wakefulness stages into others [35]. Subsequently, it leads to different parasomnia events [36, 37]. However, according to our knowledge, there are very limited data regarding the prevalence and frequency of parasomnias in the group of OSAS patients. Whether the theoretical model described above has a clinical meaning in OSAS is still to be determined. Other parasomnia risk factors also contribute to the stimulation of those mechanisms (psychoactive substances, drugs, sleep deprivation, shift-working, emotional stress, and genetic factors) [23, 38]. However, much research still needs to be performed in order to discover the exact mechanisms of sleep stage transition and the neuronal dysfunctions mentioned above.

\section{Objectives}

This study was designed as a cross-sectional study in order to estimate parasomnia presence in OSAS-suspected patients at the moment of first polygraphic evaluation. The basic goal of this study was to adapt PADSS in Poland, and to verify if it might prove useful as an additional screening tool in the group of patients with OSAS. Secondary goals included the determination of the frequency and severity of parasomnias in the group of patients with suspected OSAS. If the relation of OSAS and parasomnias really exists, the use of such a tool even for screening purpose could be of value. The aim was also to find any correlations between polygraphy-evaluated parameters and the severity and frequency of parasomnias.

\section{Material and methods}

\section{Participants}

Patients of the Department and Clinic of Internal Medicine, Occupational Diseases, Hypertension and Clinical Oncology of Wroclaw Medical University subsequently hospitalized for the assessment of possible obstructive sleep apnoea were enrolled, after receiving informed consent. Inclusion criteria consisted of adult age and suspicion of developing OSAS in patients who had not been diagnosed with OSAS before. OSAS suspicion was based on clinical symptoms and/or increased risk of OSAS shown by the STOP BANG and Berlin questionnaires. A total number of 128 patients were recruited and evaluated with polygraphy. All of them had indications for this diagnostic procedure. $\ln n=19$ patients it was impossible to get necessary data via polygraphic evaluation due to technical issues, and those patients did not come back for the second examination. $N=4$ patients refused to fill in the PADSS before the polygraphic evaluation. Finally, 105 patients were included in the statistical analysis.

All participants gave their informed consent for inclusion in the study. Polygraphy was performed in all cases under clinical conditions, outside of the study protocol. In line with the Journal's current regulations, Ethics Committee approval was not required for the inclusion period 2013-2015.

\section{Parasomnia evaluation}

Usually, almost every parasomnia is diagnosed based on medical history (REM-sleep Related Behavior Disorder needs PSG confirmation) [25]. Still, not many well described tools with verified research history are available regarding parasomnia diagnosis and screening [40]. It was difficult to obtain a single tool that would meet all criteria (range of parasomnia symptoms, simplicity and multiple verification in research history). Finally the Paris Arousal Disorder Severity Scale (PADSS) was chosen [41]. This tool was developed mainly in order to assess and evaluate the presence, severity and frequency of parasomnias with nocturnal arousals, specifically somnambulism and sleep terrors, though some items contained in the scale also refer to other parasomnias. It has been used in several studies in groups of patients presenting sleepwalking, sexsomnia and sleep terrors $[41,63]$ According to our knowledge, PADSS has not been used so far in other studies concerning OSAS patients. PADSS consists of three main parts - the first contains questions about the patient's activity during sleep and symptoms, the second determines their frequency, and the third specifies the daytime symptoms and their influence on the quality of life. Examination with the scale takes about 5-10 minutes, which is important in the case of OSAS patients as they often suffer from attention disorders. PADSS has been not used in Poland before, so translation into Polish was performed and checked with back translation done by a professional translator.

\section{Polygraphic evaluation}

All patients underwent a standardized overnight, single night polygraphy as a reference standard for the diagnosis of OSAS [42]. The following parameters were obtained: AHI (the number of apneas and hypopneas per hour of estimated sleep time), oxygen desaturation index (ODI; the number of oxygen desaturations $>3 \%$ per hour of estimated sleep time), average and minimal blood oxygen saturation and percentage of estimated sleep time below $90 \%$ and $80 \%$ oxygen saturation. Abnormal respiratory events were evaluated according to the standard criteria of the American Academy of Sleep Medicine Task Force [43]. Additionally, all patients filled out a short survey containing questions about their age, body mass and height.

\section{Statistical analysis}

Statistical analysis was performed with the Statsoft Statistica 12 software. The analysis of the results included Shapiro-Wilk testing (normality of the distribution) with histogram analysis, non-parametrical testing (the $U$ Mann-Whitney test) and correlation calculation ( $\rho$ Spearman testing).

\section{Results}

The study group finally consisted of 105 patients, 35 women $(33.3 \%)$ and 70 men (66.6\%), aged from 21 to 77 years (mean = $56, \mathrm{SD}=12,8$ ). 
Based on the polygraphy results, participants were divided into four groups according to the recommendations of the American Academy of Sleep Medicine (mild OSAS - AHI > 5, moderate $-\mathrm{AHI}>15$ or severe $-\mathrm{AHI}>30)$ : non-OSAS $(n=19,18.1 \%)$, mild OSAS $(n=31,29.52 \%)$, moderate OSAS $(n=21,20 \%)$ and severe OSAS ( $n=34,32.38 \%)$. From a clinical perspective, mild OSAS treatment includes only elimination of risk factors (mainly body mass reduction and lifestyle changes) and further observation. In these patients clinical symptoms are often very subtle or absent. Proper treatment with CPAP is recommended for some patients with moderate OSAS (if they present severe clinical symptoms like increased daytime sleepiness or cardiovascular risk factors) and all patients with severe OSAS [44, 65]. Thus, it was primarily verified if PADSS is a proper tool for diagnostics of moderate or severe OSAS.

Based on the recommended cut-off score of 13 points [41], two groups of patients emerged: with positive $(n=10)$ and negative $(n=95)$ results. Compared to the polygraphy evaluation, PADSS achieved very low sensitivity $(10.9 \%)$ and high specificity (92\%) when used for moderate and severe OSAS screening. The positive predictive value (PPV) reached $60 \%$, negative predictive value (NPV) $-48.4 \%$. Comparatively, when used for the screening of any OSAS severity level, PADSS achieved a sensitivity of $10.46 \%$ and a specificity of $94.73 \%$. The low sensitivity level eliminates the possibility of using PADSS for screening purposes with the recommended cut-off level.

Nevertheless, statistically higher PADSS scores in patients with elongation of the percentage of estimated sleep time below $80 \%$ blood oxygen saturation ( $p=0.006$, Spearman correlation $\rho=0.43$ ) were found. Weaker statistical relationships were found in minimal oxygen saturation $(p=0.097)$ and percentage of estimated sleep time below $90 \%$ blood oxygen saturation $(p=0.068)$ in patients with positive PADSS score.

Lowering the cut-off level down to 10 points did not significantly affect sensitivity $-20 \%$ in patients with moderate/severe OSAS, specificity $88 \%$ ( $n=17$ PADSS-positive patients after cut-off lowering). The statistical relationship of the percentage of estimated sleep time below $80 \%$ blood oxygen saturation in the group with positive PADSS scores was again confirmed $(p=0.03)$. A weaker trend was observed in PADSS-positive patients and AHI value $(p=0.074)$, which did not occur in the positive group with the recommended cut-off value.

Finally, participants were divided based only on the frequency of their reported parasomnia symptoms (second section of PADSS), ignoring their qualitative complaints. Group 0 contained patients who did not report any parasomnia events $(n=66)$. Patients in group I confirmed rare occurrence of symptoms, less than once a week $(n=20)$, while patients in group II reported frequency of at least once a week $(n=9)$. Group III reported parasomnia events to occur every night $(n=10)$. Parasomnia events happening at least once a week determine frequency of clinical importance, and those patients were recognized as having a positive score (groups II and III). Once again, the majority of patients still had a negative score $(n=86)$, even with the cut-off criterion changed. With this change, high specificity $(86 \%)$ with low sensitivity $(26.6 \%)$ was achieved in the group of patients with moderate/severe OSAS. The positive predictive value reached $63 \%$, whereas the negative predictive value only $50 \%$. In the group of patients with any OSAS severity level, sensitivity totalled $38 \%$ and specificity $72 \%$.

Again, some statistical relationships and trends were observed that could suggest a possible explanation of parasomnia events occurring in OSAS patients. In patients with increased percentage of estimated sleep time below $80 \%$ blood oxygen saturation the frequency of parasomnia events was significantly higher $(p=0.02$, Spearman $\rho=0.31)$. There was also a very weak trend in the relationship between minimal saturation and parasomnia frequency ( $p=0.11$, Spearman $\rho=0.16)$. Comparatively, the relationship of parasomnia frequency and $\mathrm{AHI}$ value seems much less possible $(p=0.17)$.
Also, some interesting trends were observed regarding the answers given in the third section of PADSS (referring to nocturnal episodes' consequences, which include disturbance of other people's sleep, hurting oneself or another person, fatigue, and daytime mood and functioning). Patients giving positive answers to those questions $(n=19)$ have decreased minimal blood oxygenation values and increased percentage of estimated sleep time below $80 \%$ blood oxygen saturation $(p=0.07$ and $p=$ 0.08 , respectively). Among those patients as many as 16 people belong to groups II and III (parasomnia frequency criterion). It is worth mentioning that those questions also refer to typical OSAS symptoms (fatigue, mood disorders, daytime sleepiness), so the positivity of answers may depend on apnea severity, and not parasomnia presence.

\section{Discussion}

Unfortunately, PADSS did not turn out to be useful as an additional tool for OBS diagnosis and screening. The sensitivity level, PPV and NPV were too low. It also affected the statistical analyses of our data - our tests do not have high statistical value, mainly due to the inequality of the compared groups (only 10 positive results when using the recommended cut-off level and 19 positive results using our frequency criterion). The low sensitivity could be caused by the fact that PADSS is mainly focused on the evaluation of nocturnal arousals in somnambulism and sleep terrors, while only a few questions refer to other parasomnias (like sexsomnia, enuresis, and sleep-related eating disorder). A few symptoms especially often reported by patients with OSAS are not included in PADSS (catathrenia, sleep talking and sleep paralysis), as they usually do not lead to arousals. We assume that another tool with a wider range of questions concerning different disorders could be much more sensitive in this group of patients.

Due to technical limitations, the evaluation of sleep microstructure was impossible, and this is why no data regarding this subject were presented. Changes of NREM and REM microstructure in OSAS are well known [45]. The research team is currently in an advanced stage of research concerning this issue, and is testing a new parasomnia and sleep-related movement disorders screening tool that may be valid for OSAS patients.

Probably the greatest impact of OSAS on brain function is based on cyclical intermittent hypoxia [46]. Lim describes three ways in which this process affects the blood-brain barrier (BBB) and thus brain functioning during sleep: promoting oxidative stress, inducing so-called "hypoxia inducible factors" (HIFs) in cells, and finally causing chronic inflammation in the BBB area. Oxidative stress in neurons is a known factor of neurodegeneration in many dementive diseases, and is a possible cause of neurodegeneration in long-suffering OSAS patients leading to cognitive dysfunctions $[47,48]$. Such degeneration was even observed in neuroimaging studies [49-51]. HIFs act as transcription factors in brain cells, promoting genes responsive for hypoxia reaction like glucose metabolism, erythropoietin production and angiogenesis [52-54]. Chronic inflammation in some studies seems to affect the BBB much more in OSAS patients with obesity than those with $\mathrm{BMI}<30[55,56]$. Lim and Pack describes studies that lead to the conclusion that those three processes impair the microvessel permeability of the BBB, although it is still too early to determine whether this impairment is a sufficient factor in causing neuronal morphologic changes or synaptic dysfunctions [46].

This study delivers data that somehow support this theory. We have shown a statistically significant relation of percentage of total estimated sleep time below $80 \%$ blood oxygen saturation with arousal disorder severity (measured with PADSS) in OSAS patients. We could not determine if the patients already had morphological changes in the brain without the use of neuroimaging techniques. However, the presence of parasomnia 
may suggest abnormalities in sleep microstructure on a sufficiently deep level to cause REM/NREM and wakefulness intrusions in different sleep stages that lead to parasomnia events. Nevertheless, some of the PADSS high-scoring patients did not have significantly elongated sleep time with lowered saturation level, so other factors have to contribute to parasomnia events in this group (possibly changes in sleep microstructure and arousals due to apnea events [23, 35], which were not evaluated in our study). Whether intermittent hypoxia is a sole risk factor of parasomnia is yet to be determined. It is also worth noting that we did not obtain any strong evidence that $\mathrm{AHI}$ is significantly higher in those patients. This supports the theory that AHI does not truly show the impact of OSAS on blood oxygen saturation and brain hypoxia risk. Lim and Pack came to similar conclusions [46]. Vgontzas observed that the association between $\mathrm{AHI}$ and daytime sleepiness symptoms is rather weak, and $\mathrm{AHI}$ alone may not represent the severity of OSAS [57]. Anyway, the results suggest that patients with increased $\mathrm{AHI}$ do not have statistically higher PADSS scores or increased frequency of arousal disorders.

Several studies showed that sleep fragmentation and sleep efficiency do not contribute directly to increased daytime sleepiness, and other factors have to be found in the OSAS patient group [17]. Some possible associations of daytime sleepiness, fatigue and daily mood disorders (the third section in PADSS) with minimal blood oxygen values, as well as the percentage of estimated sleep time below $80 \%$ blood oxygen saturation were observed. Such results may support the hypothesis that cyclical intermittent hypoxia may affect daytime functioning, which was previously described by Miliauskas and Sakalauskas and Jacobsen et al. $[58,59]$. What is once again worth noting is that no association between the symptoms reported in the last section of PADSS and AHI was found. So again, AHI turned out not to be an efficient marker for blood oxygen saturation evaluation. Taking into account the fact that most of the patients giving positive answers in this section (16 out of 19) belonged to groups II and III of parasomnia events frequency, we can assume that parasomnia disorders may contribute to impaired daytime functioning in OSAS patients. Zadra et al., based on several studies, came to the conclusion that somnambulism itself may be characterized by excessive daytime sleepiness, even after nights without arousal episodes [60]. Zhou et al. described several studies which stated that many patients, even with severe OSAS, do not report excessive daytime sleepiness or other daytime functioning impairments $[17,61,62]$. The results suggest that parasomnia may contribute to the further impairment of daytime functioning, which may be a priori worsened by the OSAS itself. However, our study has its limitations - the evaluation of daytime sleepiness with other tools was not performed.

Some other weaker trends that were observed (including associations between minimal oxygen saturation, percentage of sleep time below $90 \%$ blood oxygen saturation and parasomnia severity) together with the previously described results could also support the statement that parasomnia events may somehow be evoked by cortical arousals typical for OSAS superimposed on intermittent hypoxia and sleep deprivation [22]. However, the data still do not have sufficiently strong statistical power to clearly support those hypotheses, mainly due to the small number of PADSS-positive patients, and lack of EEG monitoring during polygraphic diagnostics. Further research is needed, including focusing on microstructural sleep disturbances and neuroimaging in OSAS patients with parasomnias, including tools with higher sensitivity, and possibly including other sleep complaints like sleep-related movement disorders or catathrenia [24]. It is also worth mentioning that patients with OSAS often report insomnia-like complaints, which are generally a common problem in Polish primary care [63]. This is why family doctors should always consider OSAS as a possible cause of insomnia and increased daytime sleepiness.

\section{Limitations of the study}

We are aware that this study has some limitations. Our study provides limited statistical power to confirm the hypothesis that cyclic intermittent hypoxia might be the cause of higher frequency of arousal disorders in OSAS patients. According to our knowledge, PADSS has not been validated in OSAS - our study is the first to use PADSS in this group of patients. Due to technical limitations, we were not able to assess the patients with full polysomnographic evaluation, so polygraphy was performed instead. The sample size in this study is relatively small and the results are partially under-powered. This limitation is determined due to lack of financial support, which hampered the possibility of further recruitment. Such studies very often meet some sample or time limitations, and we are aware of this fact. However, we believe that this study indicates some directions for future research, including bigger samples and better methodology (including other questionnaires and full polysomnographic evaluation), and the results obtained in this study are sufficient to justify the cautious conclusions included in the text. Currently, another study regarding parasomnias in OSAS is being carried out by this team.

\section{Conclusions}

- Due to low sensitivity, PPV and NPV PADSS cannot be used as a screening tool in possible OSAS assessment. Another tool with higher sensitivity should be developed, also including the assessment of other parasomnias and sleep-related movement disorders.

- Cyclic intermittent hypoxia might be the cause of the higher frequency of arousal disorders in OSAS patients. However, our study did not provide sufficient statistical power to fully confirm this hypothesis. Further studies concerning this topic are needed.

- High AHI value does not directly increase the frequency of arousal disorders.

Source of funding: This work was funded from the authors' own resources. Conflicts of interest: The authors declare no conflicts of interest.

\section{References}

1. Wilcox I, McNamara SG, Collins FL, et al. "Syndrome Z": the interaction of sleep apnoea, vascular risk factors and heart disease. Thorax 1998; 53(Suppl. 3): S25-S28.

2. Lavie L. Oxidative stress inflammation and endothelial dysfunction in obstructive sleep apnea. Front Biosci (Elite Ed) 2012; 4: 1391$-1403$.

3. Chervin RD. Sleepiness, fatigue, tiredness, and lack of energy in obstructive sleep apnea. Chest 2000; 118(2): 372-379.

4. Young T, Peppard PE, Gottlieb DJ. Epidemiology of obstructive sleep apnea: a population health perspective. Am J Respir Crit Care Med 2002; 165: 1217-1239.

5. Young T, Shahar E, Nieto FJ, et al. Sleep Heart Health Study Research Group. Predictors of sleep-disordered breathing in community dwelling adults: the Sleep Heart Health Study. Arch Intern Med 2002; 162: 893-900. 
6. Stradling JR, Crosby JH. Predictors and prevalence of obstructive sleep apnoea and snoring in 1001 middle aged men. Thorax 1991; 46: 85-90.

7. Peppard PE, Young T, Palta M, et al. Prospective study of the association between sleep-disordered breathing and hypertension. $N$ Engl J Med 2000; 342: 1378-1410.

8. Munoz R, Duran-Cantolla J, Martínez-Vila E, et al. Severe sleep apnea and risk of ischemic stroke in the elderly. Stroke 2006; 37 : 2317-2321.

9. Arzt M, Young T, Finn L, et al. Association of sleep-disordered breathing and the occurrence of stroke. Am J Respir Crit Care Med 2005; 172: $1447-1510$.

10. Mooe T, Franklin KA, Wiklund U, et al. Sleep-disordered breathing and myocardial ischemia in patients with coronary artery disease. Chest 2000; 117(6): 1597-1602.

11. Peled N, Abinader EG, Pillar G, et al. Nocturnal ischemic events in patients with obstructive sleep apnea syndrome and ischemic heart disease: effects of continuous positive air pressure treatment. J Am Coll Cardiol 1999; 34(6): 1744-1749.

12. Tan KC, Chow WS, Lam JC, et al. HDL dysfunction in obstructive sleep apnea. Atherosclerosis 2006; 184: 377-382.

13. Lin MT, Lin HH, Lee PL, et al. Beneficial effect of continuous positive airway pressure on lipid profiles in obstructive sleep apnea: a metaanalysis. Sleep Breath 2015; 19: 809-817.

14. Al-Delaimy WK, Manson JE, Willett WC, et al. Snoring as a risk factor for type II diabetes mellitus: a prospective study. Am J Epidemiol 2002; 155: 387-393.

15. Chen L, Pei JH, Chen HM. Effects of continuous positive airway pressure treatment on glycaemic control and insulin sensitivity in patients with obstructive sleep apnoea and type 2 diabetes: a meta-analysis. Arch Med Sci 2014; 10: 637-642.

16. Mehra R, Benjamin EJ, Shahar E, et al. Association of nocturnal arrhythmias with sleep-disordered breathing: the sleep heart health study. Am J Respir Crit Care Med 2006; 173(8): 910-916.

17. Zhou J, Camacho M, Tang X, et al. A review of neurocognitive function and obstructive sleep apnea with or without daytime sleepiness. Sleep Med 2016; 23: 99-108.

18. Vaessen TJA, Overeem S, Sitskoorn MM. Cognitive complaints in obstructive sleep apnea. Sleep Med Rev 2014; 19: 51-58.

19. Kreivi HR, Virkkula P, Lehto JT, et al. Upper airway symptoms in primary snoring and in sleep apnea. Acta Oto-Laryngol 2012; 132(5): 510-518.

20. American Academy of Sleep Medicine. European Respiratory Society. Australasian Sleep Association. American Thoracic Society. Sleeprelated breathing disorders in adults: recommendations for syndrome definition and measurement techniques in clinical research: the report of an American Academy of Sleep Medicine Task Force. Sleep 1999; 22: 667-689.

21. Thorpy MJ. Classification of sleep disorders. Neurotherapeutics 2012; 9(4): 687-701.

22. Howell MJ, Schenck CH. NREM sleep parasomnias in adults: confusional arousals, sleepwalking, sleep terrors, and sleep-related eating disorder. In: Barkoukis T, Matheson J, Ferber R, et al., eds. Therapy in sleep medicine. Elsevier Inc.; 2012: 559-572.

23. Mahowald MW, Cramer Bornemann MA. Non-REM arousal parasomnias. In: Kryger MH, Roth T, Dement WC, eds. Principles and practice of sleep medicine. 5th ed. St. Louis (MO): Saunders/Elsevier; 2011: 1075-1082.

24. American Academy of Sleep Medicine. International Classification of Sleep Disorders. Diagnostic and coding manual. 2nd ed. Westchester (IL): American Academy of Sleep Medicine; 2005.

25. American Academy of Sleep Medicine. International Classification of Sleep Disorders. 3rd ed. Darien (IL): American Academy of Sleep Medicine; 2014.

26. Mahowald MW, Schenck CH. Dissociated states of wakefulness and sleep. Neurology 1992; 42: 44-52.

27. Mahowald MW, Schenck CH. Evolving concepts of human state dissociation. Arch Ital Biol 2001; 139: 269-300.

28. Bassetti C, Vella S, Donati F, et al. SPECT during sleepwalking. Lancet 2000; 356(9228): 484-485.

29. Yuste R, MacLean JN, Smith J, et al. The cortex as a central pattern generator. Nat Rev Neurosci 2005; 6: 477-483.

30. Tassi P, Muzet A. Sleep inertia. Sleep Med Rev 2000; 4: 341-353.

31. Horner RL, Sanford LD, Pack Al, et al. Activation of a distinct arousal state immediately after spontaneous awakening from sleep. Brain Res 1997; 778: 127-134.

32. Parrino L, Halasz P, Tassinari CA, et al. CAP, epilepsy and motor events during sleep: the unifying role of arousal. Sleep Med Rev 2006; 10: 267-285.

33. Terzano MG, Parrino L, Spaggiari MC. The cyclic alternating pattern sequences in the dynamic organization of sleep. EEG Clin Neurophysiol 1988; 69: 437-447.

34. Halasz P, Ujszaszi J, Gadoros J. Are microarousals preceded by electroencephalographic slow wave synchronization precursors of confusional awakenings? Sleep 1985; 8: 231-238.

35. Pilon M, Montplaisir J, Zadra A. Precipitating factors of somnambulism: impact of sleep deprivation and forced arousals. Neurology 2008; 70(24): 2284-2290.

36. Espa F, Dauvilliers Y, Ondze B, et al. Arousal reactions in sleepwalking and night terrors in adults: the role of respiratory events. Sleep 2002; 25: 871-875.

37. Guilleminault C, Palombini L, Pelayo R, et al. Sleepwalking and sleep terrors in prepubertal children: what triggers them? Pediatrics 2003; 111: e17-e25.

38. Hori A, Hirose G. Twin studies on parasomnias. Sleep Res 1995; 24A: 324.

39. Arkin AM. Night-terrors as anomalous REM sleep component manifestation in slow-wave sleep. Waking Sleeping $1978 ; 2: 143-147$.

40. Lomeli HA, Pérez-Olmos I, Talero-Gutiérrez C, et al. Sleep evaluation scales and questionaries: a review. Actas Esp Psiquiatr 2008; 36(1): 50-59.

41. Arnulf I, Zhang B, Uguccioni G, et al. A Scale for Assessing the Severity of Arousal Disorders. Sleep 2014; 37(1): 127-136.

42. Health Quality Ontario. Polysomnography in patients with obstructive sleep apnea: an evidence-based analysis. Ont Health Technol Assess Ser 2006; 6(13): 1-38.

43. Berry RB, Budhiraja R, Gottlieb DJ, et al. Rules for scoring respiratory events in sleep: update of the 2007 AASM Manual for the Scoring of Sleep and Associated Events. Deliberations of the Sleep Apnea Definitions Task Force of the American Academy of Sleep Medicine. J Clin Sleep Med 2012; 8(5): 597-619.

44. Adult Obstructive Sleep Apnea Task Force of the American Academy of Sleep Medicine. Clinical guideline for the evaluation, management and long-term care of obstructive sleep apnea in adults. J Clin Sleep Med 2009; 5(3): 263-276.

45. Espa F, Dauvilliers Y, Ondze B, et al. Arousal reactions in sleepwalking and night terrors in adults: the role of respiratory events. Sleep 2002; 25: 871-875.

46. Lim DC, Pack Al. Obstructive sleep apnea and cognitive impairment: addressing the blood-brain barrier. Sleep Med Rev 2014; 18(1): 35-48.

47. Brown MK, Naidoo N. The UPR and the anti-oxidant response: relevance to sleep and sleep loss. Mol Neurobio/ 2010; 42: 103-113. 
48. Daulatzai MA. Evidence of neurodegeneration in obstructive sleep apnea: relationship between obstructive sleep apnea and cognitive dysfunction in the elderly. J Neurosci Res 2015; 93(12): 1778-1794.

49. Macey PM, Henderson LA, Macey KE, et al. Brain morphology associated with obstructive sleep apnea. Am J Respir Crit Care Med 2002; 166: 1382-1387.

50. Macey PM, Kumar R, Woo MA, et al. Brain structural changes in obstructive sleep apnea. Sleep 2008; 31: 967-977.

51. Morrell MJ, McRobbie DW, Quest RA, et al. Changes in brain morphology associated with obstructive sleep apnea. Sleep Med 2003; 4: 451-454.

52. Semenza GL. HIF-1: mediator of physiological and pathophysiological responses to hypoxia. J Appl Physiol 2000; 88: 1474-1480.

53. Dayyat EA, Zhang SX, Wang $Y$, et al. Exogenous erythropoietin administration attenuates intermittent hypoxia-induced cognitive deficits in a murine model of sleep apnea. BMC Neurosci 2012; 13: 77, doi: 10.1186/1471-2202-13-77.

54. Benderro GF, Lamanna JC. Hypoxia-induced angiogenesis is delayed in aging mouse brain. Brain Res 2011; 1389: 50-60.

55. Drager LF, Li J, Reinke C, et al. Intermittent hypoxia exacerbates metabolic effects of diet-induced obesity. Obesity (Silver Spring) 2011; 19: 2167-2174.

56. Arnardottir ES, Maislin G, Schwab RJ, et al. The interaction of obstructive sleep apnea and obesity on the inflammatory markers C-reactive protein and interleukin-6: the Icelandic sleep apnea cohort. Sleep 2012; 35: 921-932.

57. Vgontzas AN. Excessive daytime sleepiness in sleep apnea: it is not just apnea-hypopnea index. Sleep Med 2008; 9: 712-714.

58. Miliauskas S, Sakalauskas R. Obstructive sleep apnea: factors important for severe daytime sleepiness. Medicina (Kaunas) 2003; 39 : 232-236.

59. Jacobsen JH, Shi L, Mokhlesi B. Factors associated with excessive daytime sleepiness in patients with severe obstructive sleep apnea. Sleep Breath 2013; 17: 629-635.

60. Zadra A, Desautels A, Petit D, et al. Somnambulism: clinical aspects and pathophysiological hypotheses. Lancet Neurol 2013; 12(3): 285-294.

61. Roure N, Gomez S, Mediano O, et al. Daytime sleepiness and polysomnography in obstructive sleep apnea patients. Sleep Med 2008; 9: 727-731.

62. Rey de Castro J, Rosales-Mayor E. Clinical and polysomnographic differences between OSAH patients with/without excessive daytime sleepiness. Sleep Breath 2013; 17: 1079-1086.

63. Dudzińska M, Neć M, Zwolak A, et al. Bezsenność - istotny problem w codziennej praktyce lekarskiej - doniesienia wstępne. Fam Med Prim Care Rev 2015; 17(2): 90-93 (in Polish).

64. Dubessy AL, Leu-Semenescu S, Attali V, et al. Sexsomnia: a specialized non-REM parasomnia? Sleep 2017; 40(2), doi: 10.1093/sleep/ zsw043.

65. Pływaczewski R, Brzecka A, Bielicki P, et al. Sleep related breathing disorders in adults - recommendations of Polish Respiratory Society. Pneumonol Alergol Pol 2013; 81(3): 221-258.

Tables: 0

Figures: 0

References: 65

Received: 08.08.2017

Reviewed: 21.08.2017

Accepted: 25.10 .2017

Address for correspondence:

Patryk Piotrowski, MD, PhD

Katedra Psychiatrii UM

Wybrzeże L. Pasteura 10

50-367 Wrocław

Polska

Tel.: + 4871 784-16-00

E-mail: patryk.piotrowski@umed.wroc.pl 